\title{
Cascading failures in networks of heterogeneous node behaviour
}

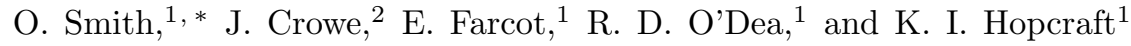 \\ ${ }^{1}$ School of Mathematical Sciences, University of Nottingham, NG7 2RD, United Kingdom \\ ${ }^{2}$ Faculty of Engineering, University of Nottingham, NG7 2RD, United Kingdom
}

\begin{abstract}
Variability in the dynamical function of nodes comprising a complex network impacts upon cascading failures that can compromise the network's ability to operate. Node types correspond to sources, sinks or passive conduits of a current flow, applicable to renewable electrical power micro-grids containing a variable number of intermittently operating generators and consumers of power. The resilience to cascading failures of ensembles of synthetic networks with different topology is examined as a function of the edge current carrying capacity and mix of node types, together with exemplar real-world networks. Whilst a network with homogeneous node type can be resilient to failure, one with identical topology but heterogeneous node function can be strongly susceptible to failure. For networks with similar numbers of sources, sinks and passive nodes the mean resilience decreases as networks become more disordered. Nevertheless all network topologies have enhanced regions of resilience, accessible by manipulation of node composition and functionality.
\end{abstract}

Naturally occurring examples of complex networks include, inter alia, transportation, economic and social structures, biological systems and power grids. When a dynamic is imposed upon these structures, their ability to operate can be compromised by a part of the network, be it an edge or node, developing a fault which then spreads in a cascade throughout the entirety [1, 2]. Catastrophic events such as blackouts in electrical grids [3], crashes in financial markets [4, 5] and the spread of congestion and overloads in the Internet and transportation networks [6], can be described as cascading failures. A threshold cascade model was introduced in [7, in which a node in a network fails with probability proportional to the number of adjacent failed nodes. It showed that networks with heterogeneous degree distributions have increased resilience. A flow based cascade model, applied to large-scale electrical grids and the Internet, was studied in [8, 9 and highlighted the vulnerability of networks whose nodes posses heterogeneous capacities and bear a load proportional to their degree. Selective pruning of a network topology was shown to help arrest cascades in 10, 11, whilst a rewiring scheme to suppress cascades was considered in [12. Cascades have been studied on large scale power grid topologies in [13, and in [14] which showed that large scale cascades are most likely to be triggered by edges in the vicinity of a network's core. Cascades have also been investigated using a continuous time model [15, oscillator networks [16, 17] and in socalled interdependent or multiplex networks [18, 19].

These studies consider networks with fixed node types and behaviour; however, 20] showed that different nodetypes affect network efficiency and redundancy, which is allied to resilience. This paper will identify regimes of resilience to cascading failure in terms of such heterogeneity of node types, be they generators, consumers or passive carriers of flow. This is motivated by the increasing proliferation of small renewable energy resources in modern electrical grids. Such micro-grids possess a variable

\footnotetext{
* oliver.smith@nottingham.ac.uk
}

number of mutable consumers and generators of power that are typically spread throughout the networks, and these intermittent sources and sinks generally have small output and consumption. This contrasts with the rigid structure of traditional power grids which contain a small number of large-capacity generators. Understanding how this increased spread in location and variability in node function affects the operation, stability and robustness of electrical networks is an important and ongoing interdisciplinary problem 21 24 that we address.

We consider networks $\mathcal{G}=(\mathcal{V}, \mathcal{E})$, with $n=|\mathcal{V}|$ nodes and $m=|\mathcal{E}|$ edges, wherein $n_{s}$ nodes are sources of flow, $n_{d}$ are sinks and the remaining $n_{p}$ are passive or empty. Each edge $e \in \mathcal{E}$ has a flow volume $F_{e}$, computed using the linearised DC power flow equation [25]

$$
F_{e}=\left\langle E_{e}, \theta\right\rangle / x_{e} .
$$

Here $x_{e}$ is the reactance of edge $e$ and $E \in \mathbb{R}^{n \times m}$ is the node-edge incidence matrix, with $E_{e}$ its $e^{\text {th }}$ column. The inner product is denoted by $\langle\cdot, \cdot\rangle$ and $\theta \in \mathbb{R}^{n}$ is the vector of node voltage phases determined from

$$
\mathcal{L} \theta=P,
$$

where $\mathcal{L}=E \operatorname{diag}(Y) E^{T}$ is the weighted graph Laplacian and $Y \in \mathbb{R}^{m}$ the vector with entries $1 / x_{e}$. The flow injection vector $P \in \mathbb{R}^{n}$ has entries

$$
P_{v}= \begin{cases}\left(1+\zeta_{v}\right) P^{\mathrm{tot}} / n_{s}, & \text { if node } v \text { is a source } \\ -\left(1+\zeta_{v}\right) P^{\mathrm{tot}} / n_{d}, & \text { if node } v \text { is a sink } \\ 0, & \text { otherwise }\end{cases}
$$

with $P^{\text {tot }}$ the total power injected into the network and $\zeta_{v}$ a random variable used to induce heterogeneity in source and sink strength.

Solutions of eqs. 12 describe a flow where supply and demand is matched, and this will be enforced throughout a cascading failure, which proceeds as follows. A maximum capacity $\alpha$ is assigned to each edge of the network and a cascade is initiated by computing the equilibrium flow from eqs. 112 and removing the most heavily 


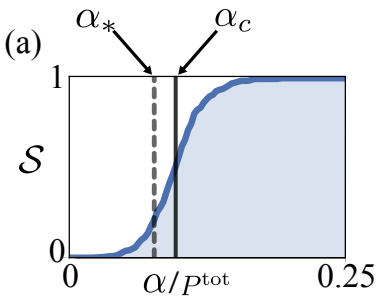

(b)

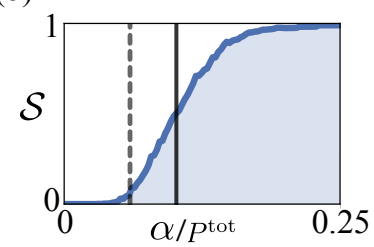

FIG. 1: The mean fraction $\mathcal{S}$ of surviving edges as a function of edge capacity $\alpha$ for ensembles of 200

Watts-Strogatz networks with $n=50, K=4, n_{s}=10$

and $n_{d}=40$. (a,b) are for $q=0.1,1$ respectively.

loaded edge, then recaculating the flow $F_{e}$ on each edge in this modified network. If $F_{e}>\alpha$ on any edge, that edge is deemed overloaded and removed. This process continues until the network attains a final equilibrium with all edges carrying a flow $\leq \alpha$. During this cascade the network may fragment into separate connected components, and so the algorithm must be recursively applied for each new connected component in which conservation of flow must be ensured. Flow surplus or deficit is prevented by adjusting source and sink strengths. We assume that sources and sinks alter their demand and supply reciprocally. If a component contains $n_{s}^{\prime}$ sources, $n_{d}^{\prime}$ sinks and a flow supply surplus $\delta$, then each source decreases its output by $\delta / 2 n_{s}^{\prime}$ and each sink increases its demand by $\delta / 2 n_{d}^{\prime}$. If the component contains either no sources or no sinks then the flow cannot be balanced and the entire component is removed. Balancing of power by non-reciprocal source/sink behaviour is discussed later, together with other models for initiating the cascade.

The network eventually attains a final state, with the fraction of surviving edges relative to the original number denoted by $\mathcal{S}$. A value of $\mathcal{S}=0$ indicates an entire network failure; $\mathcal{S}=1$ denotes complete resilience. To assess the resilience of networks to failure, $\mathcal{S}$ is computed as a function of $\alpha$, as displayed in Fig. 1(a,b) for an ensemble of 200 random networks generated by the Watts-Strogatz method [26]. These are of size $n=50$, comprising $n_{s}=10$ and $n_{d}=40$ nodes, with locations chosen uniformly at random, and chosen rewiring parameters $q=0.1,1$ characteristic of small-world and Poisson networks respectively. Both networks are susceptible to substantial disruption for capacities $\alpha \lesssim 0.06$ and are essentially robust for $\alpha \gtrsim 0.2$. Half of the network survives at the critical value $\alpha_{c}$, for which $\mathcal{S}\left(\alpha_{c}\right)=1 / 2$ : the smaller $\alpha_{c}$ is, the more resilient the network will be to failure. Fig. 1 shows that $\alpha_{c}$ increases as the networks become more Poissonian. To operate non-trivially the network must initially contain some non-zero capacity $\alpha_{*}$, and this is defined from the initial flow configuration $F_{0}$ to be $\alpha_{*}=\max \left(F_{0}\right)$. The ensemble average values of $\alpha_{*}$ are also shown in Figs. 1 $1(\mathrm{a}, \mathrm{b})$.

The fraction of surviving edges $\mathcal{S}$ depends on network size, topology, and both composition and location of the node types. To gain insight into this dependence it is instructive to consider an exemplar network of $n$ nodes with a simple ring-structure, such as that illustrated in Fig. 2(a) that has $n_{s}=2, n_{d}=n-2$, common degree $\bar{K}=2$, and shortest path distance between the two sources $d$. The dependence of $\mathcal{S}$ on the configuration space of $\alpha / \alpha_{*}$ and $d$ is shown in Fig. 2(b), within which distinct regions with defined boundaries can be identified analytically [27], as overlaid in the figure. Crucially for $\alpha / \alpha_{*} \geqslant 2$, this network is resilient to cascading failures independent of the relative location of the sources, whereas for $\alpha / \alpha_{*}<2$ the network is less robust with resilience conditional on the flow capacity and $d$. For networks of larger degree $K$ these conditional boundaries erode due to the multiple paths along which the flow can equilibrate, as shown in Fig. 2(c). Nevertheless, the resilience boundary remains robust, being independent of $d$ and occurring where $\alpha / \alpha_{*} \approx K /(K-1)$ [27. This can be understood by considering the ring-network with common degree $K$ when $n_{s}=1, n_{d}=n-1$, with initial flow apportioned equally across the $K$ edges emanating from the single source. These edges are the most heavily loaded and so $\alpha_{*} \approx P^{\text {tot }} / K$. When one of these edges is removed to initiate a cascade, the flow is redistributed among the $K-1$ remaining edges connected to the source and these must now supply flow to the rest of the network. Consequently $\alpha_{c} \approx P^{\text {tot }} /(K-1)$ and therefore $\alpha_{c} / \alpha_{*} \approx K /(K-1)$. This relationship holds for larger networks, as shown in Fig. 2(d). The observed resilience boundary prompts defining a metric $\rho$ to be

$$
\rho=\frac{\alpha_{c}}{\alpha_{*}} \equiv \frac{S^{-1}(1 / 2)}{\max \left(F_{0}\right)},
$$

which gauges the relative increase in edge capacity required to survive a cascade. The smaller the value of $\rho$ the more resilient is the network.

For ensembles of random networks $\rho$ does not take a single value but rather is a random variable. The approximation for $\rho$ when $n_{s}=2, n_{d}=n-2$ is replaced by $\bar{\rho} \approx \bar{K} /(\bar{K}-1)$, where $\bar{K}$ is the mean degree that depends on $n$ and $q$ as shown in Fig. 3(a). For Watts-Strogatz networks the probability density $P(\rho)$ is log-normal with mean value $\bar{\rho}$ that scales with network size $n$ but with similar standard deviation $\sigma \approx 0.255$ across the full range of rewiring parameters $q \in[0,1]$ that encompass small-world to Poisson network topologies. This data collapse is illustrated in Fig. 3. (b) (i,ii) for $q=0.1,0.6$, respectively; panel (b)(iii,iv) shows $P(\rho)$ for two scale-free networks that have power-law degree distribution with initial node degree $m_{0}=3$, 4 , which are generated using the Albert-Barabasi preferential attachment method 28]. The range of $\rho$ is smaller than that found for WattsStrogatz networks, thereby indicating a greater resilience, and this agrees with earlier studies predicated on random edge removal [29, 30]. When $n_{s} \approx n_{d}$ the distribution is bimodal, with the larger component of the distribution corresponding to when the edge initiating the cascade is connected to a hub. Networks grown by preferential attachment, such as social networks and the Internet, pos- 
(a)

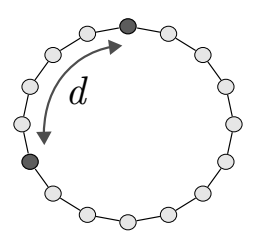

(c)

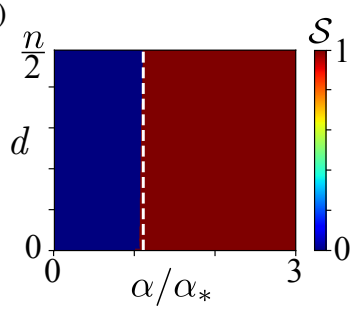

(b)

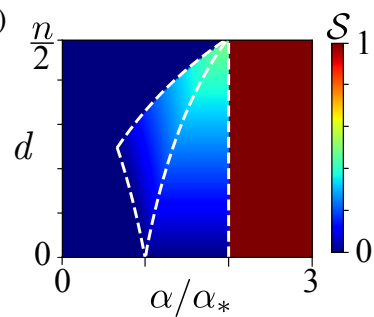

(d)

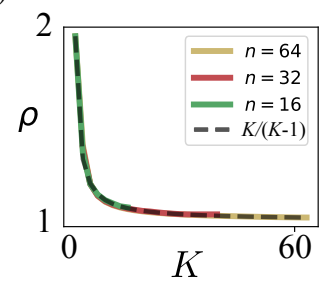

FIG. 2: (a) Periodic ring-lattice with $K=2$ containing $n_{s}=2$ sources and $n_{d}=n-2$ sinks with source separation $d$. (b) $\mathcal{S}\left(\alpha / \alpha_{*}, d\right)$ for $K=2$. (c) $\mathcal{S}$ for $K=10$. (d) $\rho(K)$ for periodic ring-lattices of different sizes, with composition $n_{s}=1$ and $n_{d}=n-1$, showing the approximation $K /(K-1)$.

sess a scale-free structure, whereas electrical grids and road/rail transportation networks do not because their structures are often dictated by geographical constraints. This paper is concerned chiefly with electrical grids, so from here networks generated by the Watts-Strogatz procedure shall provide the structural substrate on which general results regarding resilience to failures can be deduced.

(a)

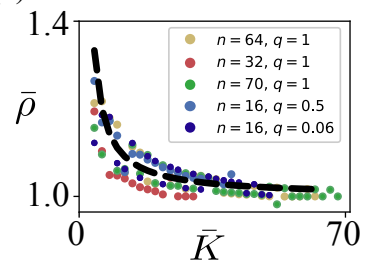

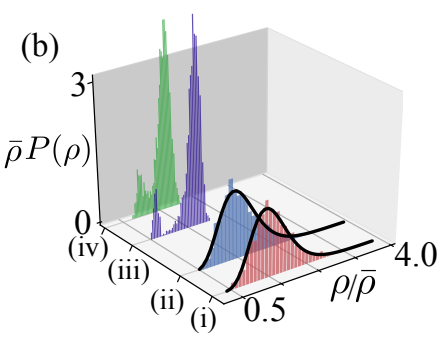

FIG. 3: Variation of $\rho$ in ensembles of 2000 random networks. (a) $\bar{\rho}(\bar{K})$ in Watts-Strogatz networks of size $n$ and rewiring parameter $q$, with $n_{s}=2, n_{d}=n-2$. The dashed line is $\bar{K} /(\bar{K}-1)$. (b) $P(\rho)$ in random networks with $\left(n_{s}, n_{d}, n_{p}\right)=(30,30,0)$. (i) $q=0.1$ and (ii) $q=0.6$. Both are fitted to $\operatorname{Lognormal}\left(\mu, \sigma^{2}\right)$ with $\mu=1.18,1.63$, and $\sigma=0.25,0.26$ respectively and confirmed with Kolmogorov-Smirnov tests. (iii) and (iv) are for scale-free networks with $m_{0}=3, m=4$ respectively.

To explore the dependence of $\rho$ on the composition of node types, note that the condition $n_{s}+n_{d}+n_{p}=n$ constrains the space of possible node configurations to a triangular simplex as illustrated in Fig. 4(a). The length of an edge of the simplex is the size $n$ of the network and any point on the triangle represents a network with a unique node-type composition; the dot in the figure represents $\left(n_{s}, n_{d}, n_{p}\right)=(5,10,5)$. Selecting a network with given $(q, K, n)$, and node-types according to $\left(n_{s}, n_{d}, n_{p}\right)$ that are located uniformly at random, the mean resilience $\bar{\rho}$ is calculated from 200 realisations and its value projected onto the corresponding location on the simplex.

Figure $4(\mathrm{~b})$ shows $\bar{\rho}$ for regular lattices (i.e. $q=0$ ) with $n=100, K=4$. The largest values of $\bar{\rho}$ and therefore the lowest resilience are located down two edges of the simplex, corresponding to networks with large numbers of sinks and very few sources, or vice versa. This means that the few source (or sink) nodes must generate (or absorb) the flow, and so incident edges must carry large flow volumes, making the network susceptible to failure. Resilient networks are spread throughout the interior region, which agrees with the intuition that a mix of node-types has a high degree of redundancy and is therefore resilient because of the multiple flow paths such networks contain.

As $q$ increases and the network structure becomes more random the morphology of $\bar{\rho}$ changes, the trend being shown in Figs. $4(\mathrm{c}-\mathrm{e})$. A band of greatest $\bar{\rho}$ broadens from the edges, moving into the interior region, until for $q \approx 0.12$ the largest values of $\bar{\rho}$ are found in the interior of the simplex. This represents an inversion in the resilience measure, counter-intuitively showing that for networks in this regime of $q$ a more heterogeneous mix of nodetypes give less resilience than does a network with a few large suppliers of power. This behaviour of $\bar{\rho}$ continues progressively until $q=1$ (a Poisson network), where $\bar{\rho}$ has largest values in the centre of the simplex and lowest values along two edges. In all cases the morphology of $\bar{\rho}$ is symmetric about the centre line, reflecting the reciprocity between sources and sinks.

The inversion in the behaviour of $\bar{\rho}$ with $q$ can be gauged by determining the value of $\Delta \rho=\bar{\rho}_{\text {Left }}-\bar{\rho}_{\text {Centre }}$, where $\bar{\rho}_{\text {Left }}$ and $\bar{\rho}_{\text {Centre }}$ are the values of $\bar{\rho}$ at the bottomleft and centre of the simplex respectively. For regular lattices $\Delta \rho>0$, whereas for Poisson networks $\Delta \rho<0$. Figure 5(a) shows that for networks with $n=60, \Delta \rho(q)$ decreases monotonically, the inversion occurring at the value of $q$ where the network transitions from approximate order to the small-world regime. Figure 5(b) shows the dependence of $\Delta \rho$ with $q$ and $n ; \Delta \rho=0$ is shown by the line and denotes the locus of the inversion, which is approximately independent of network size.

Preceding results consider homogeneous flow injection (i.e. $\zeta_{v}=0$ in eq. (3) ) and edge capacity, reciprocal source-sink behaviour, and cascades triggered by the removal of the most heavily loaded edge, which serve as a model for overloading failure. The impact of varying these model choices, and thus introducing several different types of heterogeneity, will now be considered. Cascades may be triggered by other unpredictable events such as lightening strikes or software failures and, moreover, generator output and consumer usage will vary. The behaviours and symmetry properties highlighted 

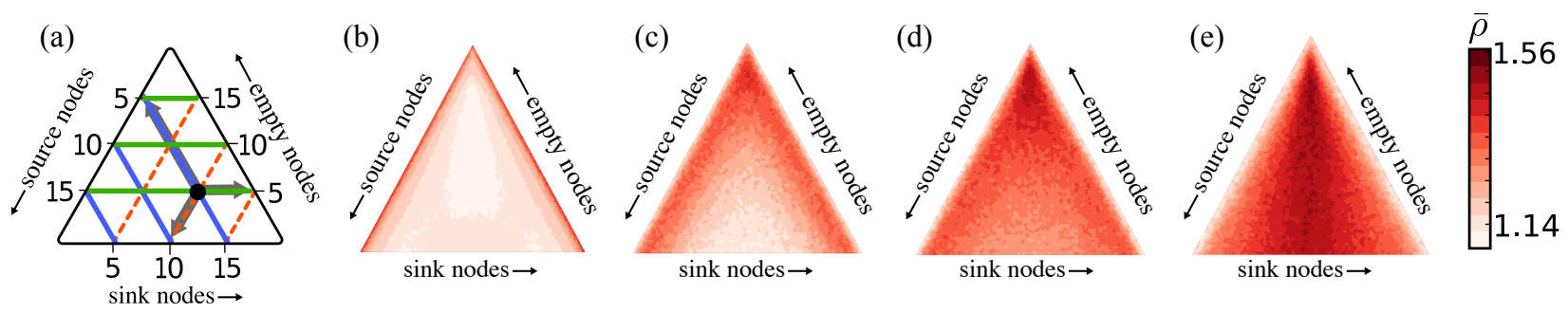

FIG. 4: (a) A sketch of the node configuration space simplex. The black dot represents a configuration of $\left(n_{s}, n_{d}, n_{p}\right)=(5,10,5)$. (b-e) $\bar{\rho}$ projected onto the simplex for networks of size $n=100, K=4$ and with $q=0,0.2,0.4$ and 1 respectively.
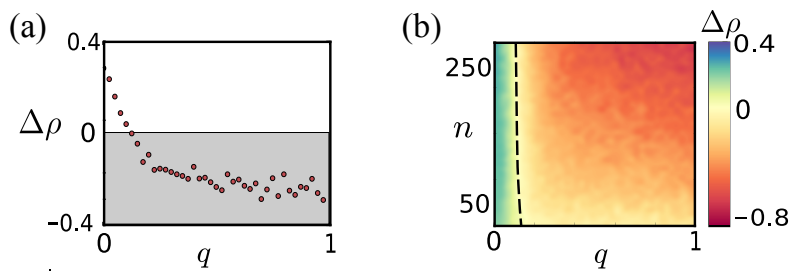

FIG. 5: (a) $\Delta \rho(q)$ for $n=60$. (b) $\Delta \rho(q, n)$ where the dashed line is the locus of the inversion $\Delta \rho=0$. All data points averaged over 200 realisations.

above persist if the cascade is initiated with a probability proportional to its initial load [27] and/or if the flow injection is perturbed by normally distributed noise with zero mean and the standard deviation selected to model generator variability, whilst still preserving node type in all realisations that we consider. These behaviours also persist for sources and sinks with gamma distributed strengths; see [27. Unlike [8, 10, this paper has considered networks whose edge capacities are homogeneous. This choice is motivated by smaller scale power networks that form our primary motivation, and whose dominant source of heterogeneity is node behaviour rather than edge capacity. Nonetheless, the impact of edge capacity heterogeneity is investigated by perturbing $\alpha$ with Gaussian noise. These results, shown in [27], demonstrate that the features observed in the homogeneous case persist. However, for networks wherein source-sink reciprocity is broken, the morphology of the simplex plots is likewise no longer symmetric. This is illustrated in 27] for the case when only sink nodes have the ability to adjust their flow output to match supply and demand.

The resilience of the Austrian national grid is considered in Fig.6.(b). This network comprises $n=67, m=85$ and $\bar{K}=2.53$ where each node represents a sub-network of node-types and edges at both meso- and micro-scales that are unresolved by the data of [31. Figure 6(b) shows $\bar{\rho}$ with each pixel entry averaged from 100 realisations of node-type location and with the sources and sinks assumed to have reciprocal strengths. The resilience landscape is characteristic of a state intermediate of those shown in Figs 4 (c) and (d). 27] includes two real-world examples of power grids which conform to the archetypes
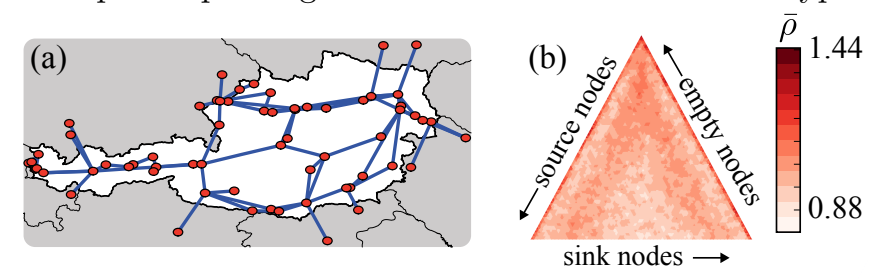

FIG. 6: (a) Power grid topology of Austria, constructed using data from 31. (b) $\bar{\rho}$ for this grid projected onto the node configuration simplex. Each pixel is an ensemble average over 100 source-sink locations.

in Fig. 4 .

This paper has considered how the resilience of networks to cascading failures is affected by the composition of node-types in addition to their topology. For the case of grids comprising a few large generators Fig. 33(a) shows that the resilience can be approximated by $\bar{K} /(\bar{K}-1)$, where $\bar{K}=\bar{K}(q, n)$. For the case of renewable energy electrical-grids, the resilience is found using simplexes of the type shown in Fig 4 . Here the appropriate node-types correspond to locations of passivity, generation or consumption of power, and these functions will mutate diurnally in response to changes in demand 24]. Crucially, although networks with homogeneous node-type of a given topology can be resilient to failure, this is no longer the case for a network of the same topology but with a heterogeneous composition of node-types. Regular lattices and ordered networks are most resilient when the number of node-types are similar. This changes as network's structure becomes increasingly disordered, with the least resilient configurations becoming those with similar numbers of node-types. Despite this trend, there still exist regimes of resilience with a range of node-type compositions that can be accessed for highly randomised network structures.

Acknowledgments OS acknowledges support from the Leverhulme Trust via the doctoral scholarship programme 'Modelling and Analytics for a Sustainable Society'. The authors are grateful for access to the University of Nottingham High Performance Computing Facility. 
[1] S. H. Strogatz, Nature 410, 268 (2001).

[2] R. Albert and A.-L. Barabási, Rev. Mod. Phys. 74, 47 (2002)

[3] B. A. Carreras, V. E. Lynch, I. Dobson, and D. E. Newman, Chaos 12, 985 (2002).

[4] X. Huang, I. Vodenska, S. Havlin, and H. E. Stanley, Sci. Rep. 3, 1219 (2013).

[5] A. G. Haldane and R. M. May, Nature 469, 351 (2011).

[6] P. Crucitti, V. Latora, and M. Marchiori, Phys. Rev. E 69, 045104 (2004).

[7] D. J. Watts, Proc. Nat. Acad. Sci. USA 99, 5766 (2002)

[8] A. Motter and Y.-C. Lai, Phys. Rev. E 66, 065102 (2003)

[9] Y.-C. Lai, A. E. Motter, and T. Nishikawa, "Complex networks," (Springer Berlin Heidelberg, Berlin, Heidelberg, 2004) pp. 299-310.

[10] A. E. Motter, Phys. Rev. Lett. 93, 098701 (2004).

[11] D. Witthaut and M. Timme, Phys. Rev. E 92, 032809 (2015)

[12] C. D. Brummitt, R. M. D'Souza, and E. A. Leicht, Proc. Nat. Acad. Sci. USA 109, E680 (2012).

[13] S. Pahwa, C. Scoglio, and A. Scala, Sci. Rep. 4, 3694 (2014)

[14] Y. Yang, T. Nishikawa, and A. E. Motter, Science 358 (2017).

[15] Y. Yang and A. E. Motter, Phys. Rev. Lett. 119, 248302 (2017)

[16] B. Schäfer, D. Witthaut, M. Timme, and V. Latora, Nat. Commun. 9, 1975 (2018).

[17] M. Rohden, D. Jung, S. Tamrakar, and S. Kettemann,
Phys. Rev. E 94, 032209 (2016).

[18] Y. Zhang, A. Arenas, and O. Yağan, Phys. Rev. E 97, 022307 (2018)

[19] S. Buldyrev, R. Parshani, G. Paul, H. Stanley, and S. Havlin, Nature 464, 1025 (2010).

[20] O. Smith, J. Crowe, R. O'Dea, and K. Hopcraft, Europhys. Lett. 127, 18001 (2019).

[21] R. M. D'Souza, Science 358, 860 (2017)

[22] A. Halu, A. Scala, A. Khiyami, and M. C. González, Sci. Adv. 2 (2016).

[23] D. E. Olivares, A. Mehrizi-Sani, A. H. Etemadi, C. A. Caizares, R. Iravani, M. Kazerani, A. H. Hajimiragha, O. Gomis-Bellmunt, M. Saeedifard, R. Palma-Behnke, G. A. Jimnez-Estvez, and N. D. Hatziargyriou, IEEE Trans. Smart Grid 5, 1905 (2014).

[24] S. Parhizi, H. Lotfi, A. Khodaei, and S. Bahramirad, IEEE Access 3, 890 (2015).

[25] B. Stott, J. Jardim, and O. Alsac, IEEE Trans. Power Systems 24, 1290 (2009).

[26] D. J. Watts and S. H. Strogatz, Nature 393, 440 (1998).

[27] See Supplemental Material at [URL will be inserted by publisher] for [analytic results and further data].

[28] A.-L. Barabasi and R. Albert, Science 286, 509 (1999)

[29] D. S. Callaway, M. E. J. Newman, S. H. Strogatz, and D. J. Watts, Phys. Rev. Lett. 85, 5468 (2000).

[30] R. Albert, H. Jeong, and A.-L. Barabási, Nature 406, 378 (2000).

[31] APG, https://www.apg.at/en/Stromnetz accessed: 2019-05-17. 